\title{
Reliable Approach for EM Scattering Calculation from Sea Surface Covered with Foams Based on MFBM and VRT Theory
}

\author{
Tao Song $(\mathbb{D}$, Chuang-Ming Tong $(\mathbb{D}$, Li-Li Cong, and Peng Peng $\mathbb{D}$ \\ Air Force Engineering University, Xian 710051, China \\ Correspondence should be addressed to Tao Song; chinataoguo1990@163.com
}

Received 4 December 2017; Accepted 24 January 2018; Published 19 February 2018

Academic Editor: Milica Rančić

Copyright (C) 2018 Tao Song et al. This is an open access article distributed under the Creative Commons Attribution License, which permits unrestricted use, distribution, and reproduction in any medium, provided the original work is properly cited.

A reliable approach based on modified facet-based model and Vector Radiative Transfer theory is presented to calculate electromagnetic scattering from a particular electrically large sea surface superimposed with foams, which can handily give both monostatic and bistatic scattering results and could be applied to synthetic aperture radar imagery simulation. The facet model is derived from Fuks' first-order small perturbation method function, and then the Kirchhoff model is introduced to revise the results in view of the dependency on cut-off wave number at near vertical incidence angles. Additionally, the contributions of foams are taken into consideration on the basis of Vector Radiative Transfer theory. The accuracy and superiority of this proposed approach are demonstrated in comparison with traditional facet model, which illustrates that the results of this approach agree better with experimental results. Moreover, several examples are given to verify that the proposed approach is of more significance at large incidence angles and high wind speed.

\section{Introduction}

Investigation on electromagnetic (EM) scattering from maritime scene is of great value in both civil and military applications, such as maritime environment monitoring and clutter rejection in target detection. Extensive endeavors have been devoted to evaluate the scattering characteristic in either theoretical or experimental terms. Usually, approaches for EM scattering calculation from rough sea surface in theoretical term can be classified as numerical category and analytical category. Among the numerical efforts, some recent attempts could be noted, for instance, the Multiple Sweep Method of Moments [1], the Accelerated ForwardBackward Method [2], and the Ordered Multiple Method [3]. Although these methods prevail over traditional numerical ones in terms of accelerated skills, they would still be too tricky and time-consuming for general applications, particularly for electrically large maritime scene. On the other hand, analytical methods have received more and more attention in view of their high efficiency, relative accuracy, and clear physical interpretation. Crombie firstly discovered the Bragg resonant phenomena that the Bragg waves resonant with EM waves dominate in the echo [4]. And then two-scale theory has been always a famous approach since it was presented in the 1970s [5]. Further, the modified two-scale method was presented and validated with better accuracy [6]. However, it is still a statistic approach, which obtains an average of the scattering coefficients under different conditions without a particular sea height map, and nothing is said about local information.

In past decades, synthetic aperture radar (SAR) has been greatly developed in consideration of its independency on daylight and weather, and SAR imaging has become one of the most important means for remote sensing of maritime environment. At the same time, local information is useful and desirable in SAR imagery simulation of marine scene, especially the one with oil film and ship wake. This demand has encouraged the development of facet-based approaches, which try to break the surface into facets and then the scattering contributions from individual facets could be obtained. A facet backscattering model was presented by Franceschetti et al. by means of Kirchhoff approximation [7], but it loses sight of the Bragg phenomena. Chen et al. came up with a slope-determined facet model that could reflect both the specular and diffuse configurations in monostatic or bistatic system [8]. 
In reality, with the increase of wind speed above sea surface, foams would come into being. What is more, onethird of sea surface would be covered with foams when the velocity of wind comes up to $25 \mathrm{~m} / \mathrm{s}$. So it is of great significance to take the influences of foams on sea surface scattering into account at high wind speed condition. Jin [9] endeavored to investigate the scattering characteristics of sea surface with foams by introducing Vector Radiative Transfer (VRT) theory into two-scale model (TSM). Liang and Guo [10] combined VRT theory with modified TSM. However, the two aforementioned statistical methods cannot be applied to SAR imagery simulation because of the deficiency of local information.

In this work, a facet-based model derived from Fuks' firstorder small perturbation method function $[11,12]$ is proposed to calculate the scattering contribution from individual sea surface facets in both monostatic and bistatic configurations. Moreover, the Kirchhoff model is introduced to revise the results because the small perturbation method is not suitable and the results are dependent on cut-off wave number at near vertical incidence angles. Meanwhile, the scattering effects of foams are considered and calculated according to the VRT theory, which is of significance at high wind speed and grazing incidence consideration. The proposed approach would be attractive on the ground that the formulation is tractable and time-saving, and the mechanism is more comprehensive including both specular and diffuse features. What is more, as this scheme can calculate the local scattering contributions, it could be readily applied to SAR imagery simulation and the analysis of scattering contributions from complex sea surface with ship wake and oil film.

\section{Model Description and Formulation}

2.1. Modified Facet-Based Model. Sea surface can be seen as a two-scale model where small-scale waves are superimposed on the large-scale waves. The ELH spectrum [13] can involve both long and short wind-driven waves, so it is used to generate sea surface here. Then the sea surface is decomposed into facets with different slopes and the total scattering can be calculated by summing up the contributions from all the facets. Therefore, an adequate formula for scattering from arbitrary slope facet needs to be derived.

As shown in Figure 1, it is assumed that the mean of the microscopic rough surface is $\zeta=0$ and the distribution satisfies statistically spatial homogeneity. When considering a unit incident plane wave $E i$ in $x o z$ plane, we can get the scattering coefficient according to the first-order small perturbation method function presented by Fuks [11, 12]:

$$
\sigma_{p q}^{0}\left(\widehat{\mathbf{k}}_{i}, \widehat{\mathbf{k}}_{s}\right)=\pi k^{4}|\varepsilon-1|^{2}\left|F_{p q}\right|^{2} S_{\zeta}\left(\mathbf{q}_{l}\right)
$$

where $\widehat{\mathbf{k}}_{i}$ is a unit vector directed from the transmitter, while $\widehat{\mathbf{k}}_{s}$ is a unit vector directed to the reception point; $\mathbf{q}_{l}$ is the projection vector of $\mathbf{q}$ on the plane $z=0 ; \mathbf{q}=\left(\widehat{\mathbf{k}}_{s}-\widehat{\mathbf{k}}_{i}\right)$; $k$ denotes the electromagnetic wave number; $\varepsilon$ indicates the relative permittivity of the dielectric surface; $p, q=h, v$ stand for the polarization direction of scattering and incident wave

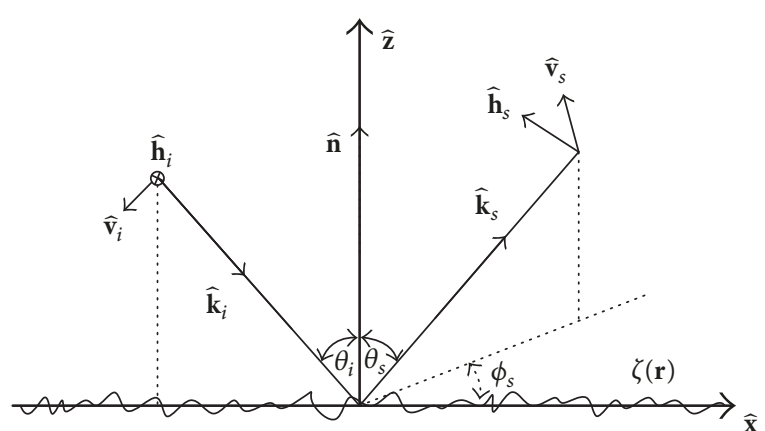

FIGURE 1: Geometry of microscopic rough scattering surface.

vector, respectively; $F_{p q}$ represents the polarization factor and detailed expressions in Appendix; $S_{\zeta}\left(\mathbf{q}_{l}\right)$ is the spatial power spectrum of microscopic rough surface.

Next, we need to calculate the scattering coefficient of arbitrary slope facet.

As shown in Figure 2, $\left\{\widehat{\mathbf{x}}_{g}, \widehat{\mathbf{y}}_{g}, \widehat{\mathbf{z}}_{g}\right\}$ is the global coordinate, while the local coordinate is created at the point $o^{\prime}$ of facet as follows:

$$
\begin{aligned}
& \widehat{\mathbf{z}}_{l}=\widehat{\mathbf{n}}, \\
& \widehat{\mathbf{y}}_{l}=\widehat{\mathbf{n}} \times \frac{\widehat{\mathbf{k}}_{i}}{\left|\widehat{\mathbf{n}} \times \widehat{\mathbf{k}}_{i}\right|}, \\
& \widehat{\mathbf{x}}_{l}=\widehat{\mathbf{y}}_{l} \times \widehat{\mathbf{z}}_{l},
\end{aligned}
$$

where $\widehat{\mathbf{n}}=\left(-Z_{x} \widehat{\mathbf{x}}_{g}-Z_{y} \widehat{\mathbf{y}}_{g}-\widehat{\mathbf{z}}_{g}\right) / \sqrt{1+Z_{x}^{2}+Z_{y}^{2}}$ indicates the normal vector of the facet; $\left(\theta_{i}, \theta_{s}, \emptyset_{i}, \emptyset_{s}\right)$ and $\left(\theta_{i}^{l}, \theta_{s}^{l}, \emptyset_{i}^{l}, \emptyset_{s}^{l}\right)$ are the global and local angles of incidence and scattering direction in different coordinates.

Referring to (1), we can write the scattering coefficient of arbitrary slope rough facet:

$$
\sigma_{P Q}^{\text {facet }}\left(\widehat{\mathbf{k}}_{i}, \widehat{\mathbf{k}}_{s}\right)=\pi k^{4}|\varepsilon-1|^{2}\left|\widetilde{F}_{P Q}\right|^{2} S_{\zeta}\left(\mathbf{q}_{l}\right),
$$

where $\widetilde{F}_{P Q}$ is defined as the polarization factor in global coordinate and can be obtained from the following relation:

$$
\begin{aligned}
& \left(\begin{array}{ll}
\widetilde{F}_{V V} & \widetilde{F}_{V H} \\
\widetilde{F}_{H V} & \widetilde{F}_{H H}
\end{array}\right) \\
& =\left(\begin{array}{cc}
\widehat{\mathbf{V}}_{s} \cdot \widehat{\mathbf{v}}_{s} & \widehat{\mathbf{H}}_{s} \cdot \widehat{\mathbf{v}}_{s} \\
\widehat{\mathbf{V}}_{s} \cdot \widehat{\mathbf{h}}_{s} & \widehat{\mathbf{H}}_{s} \cdot \widehat{\mathbf{h}}_{s}
\end{array}\right)\left(\begin{array}{ll}
F_{v v} & F_{v h} \\
F_{h v} & F_{h h}
\end{array}\right)\left(\begin{array}{cc}
\widehat{\mathbf{V}}_{i} \cdot \widehat{\mathbf{v}}_{i} & \widehat{\mathbf{V}}_{i} \cdot \widehat{\mathbf{h}}_{i} \\
\widehat{\mathbf{H}}_{i} \cdot \widehat{\mathbf{v}}_{i} & \widehat{\mathbf{H}}_{i} \cdot \widehat{\mathbf{h}}_{i}
\end{array}\right),
\end{aligned}
$$

where $\left(\widehat{\mathbf{H}}_{i}, \widehat{\mathbf{V}}_{i}, \widehat{\mathbf{H}}_{s}, \widehat{\mathbf{V}}_{s}\right)$ and $\left(\widehat{\mathbf{h}}_{i}, \widehat{\mathbf{v}}_{i}, \widehat{\mathbf{h}}_{s}, \widehat{\mathbf{v}}_{s}\right)$ are the unit polarization vectors in global and local coordinate, respectively.

According to the Bragg resonance hypothesis, both the Bragg waves spreading along and against the radar sight direction contribute to the radar receiver; thus (3) can be rewritten as

$$
\sigma_{P Q}^{\text {facet }}\left(\widehat{\mathbf{k}}_{i}, \widehat{\mathbf{k}}_{s}\right)=\pi k^{4}|\varepsilon-1|^{2}\left|\widetilde{F}_{P Q}\right|^{2} S_{\text {capi }}\left(\mathbf{q}_{l}\right),
$$




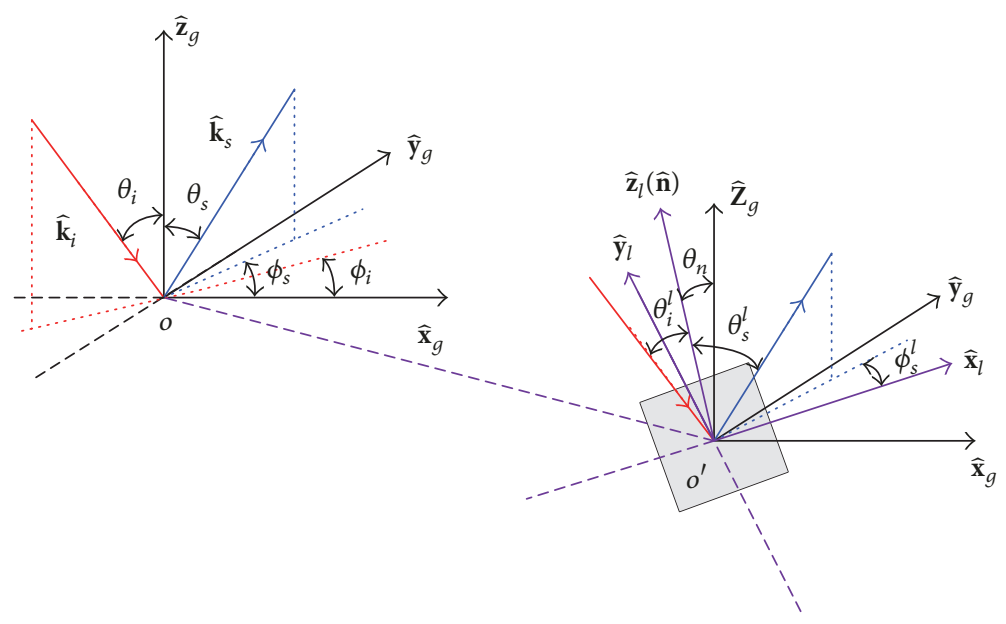

FIGURE 2: Geometry of global coordinate and local coordinate.

where $S_{\text {capi }}\left(\mathbf{q}_{l}\right)=(1 / 2)\left[S_{E}^{\text {capi }}\left(-\mathbf{q}_{l}\right)+S_{E}^{\text {capi }}\left(\mathbf{q}_{l}\right)\right]$ is the Bragg waves component; $S_{E}^{\text {capi }}$ is the small-scale capillary ripple part of one-sided ELH spectrum.

Thus, cut-off wave number $k_{\text {cut }}$ is introduced to divide ELH spectrum into large-scale gravity wave part $S_{E}^{\text {grav }}$ and small-scale capillary ripple part $S_{E}^{\text {capi }}$ :

$$
\begin{aligned}
& S_{E}^{\text {grav }}(\mathbf{k})= \begin{cases}0, & |\mathbf{k}| \geq \frac{\pi}{6} \\
S_{E}(\mathbf{k}), & |\mathbf{k}| \leq \frac{\pi}{6}\end{cases} \\
& S_{E}^{\text {capi }}(\mathbf{k})= \begin{cases}S_{E}(\mathbf{k}), & |\mathbf{k}| \geq \frac{\pi}{6} \\
0, & |\mathbf{k}| \leq \frac{\pi}{6},\end{cases}
\end{aligned}
$$

where $S_{E}(\mathbf{k})$ is the two-dimensional ELH spectrum and the $k_{\text {cut }}$ here is equal to $\pi / 6$.

Assume that the lengths of the two-dimensional simulation sea surface sample in $x$ and $y$ directions are $L_{x}$ and $L_{y}$, respectively. The area is $A=L_{x} L_{y}$, the numbers of discrete points are $M$ and $N$, and the distances between two adjacent points are $\Delta x$ and $\Delta y$. Then, according to (5), we can get the total scattering coefficient by summing up the contributions from all of the facets:

$$
\sigma_{P Q}^{\text {total }}\left(\widehat{\mathbf{k}}_{i}, \widehat{\mathbf{k}}_{s}\right)=\frac{1}{A} \sum_{m=1}^{M} \sum_{n=1}^{N}\left[\sigma_{P Q, m n}^{\mathrm{TSPM}}\left(\widehat{\mathbf{k}}_{i}, \widehat{\mathbf{k}}_{s}\right) \Delta x \Delta y\right]
$$

where $\sigma_{P Q, m n}^{\mathrm{TSPM}}\left(\widehat{\mathbf{k}}_{i}, \widehat{\mathbf{k}}_{s}\right)$ is the normalized Bragg scattering coefficient of the number $m n$ facet.

Unfortunately, there is no unified standard to choose the advisable cut-off wave number. As shown in Figure 3, the small perturbation method is not suitable and the results are dependent on $k_{\text {cut }}$ at near vertical incident angles.

The parameters in the simulation are in order as follows: the incident wave is at $\mathrm{Ku}$-band $(14.0 \mathrm{GHz})$, the wind speed at $10 \mathrm{~m}$ above the mean sea level is selected by $u_{10}=5.0 \mathrm{~m} / \mathrm{s}$, the angle of wind direction $\emptyset_{w}$ is defined as the included angle of wind direction and $\widehat{\mathbf{x}}_{g}$ axis, here $\emptyset_{w}=0^{\circ}$, the numbers of

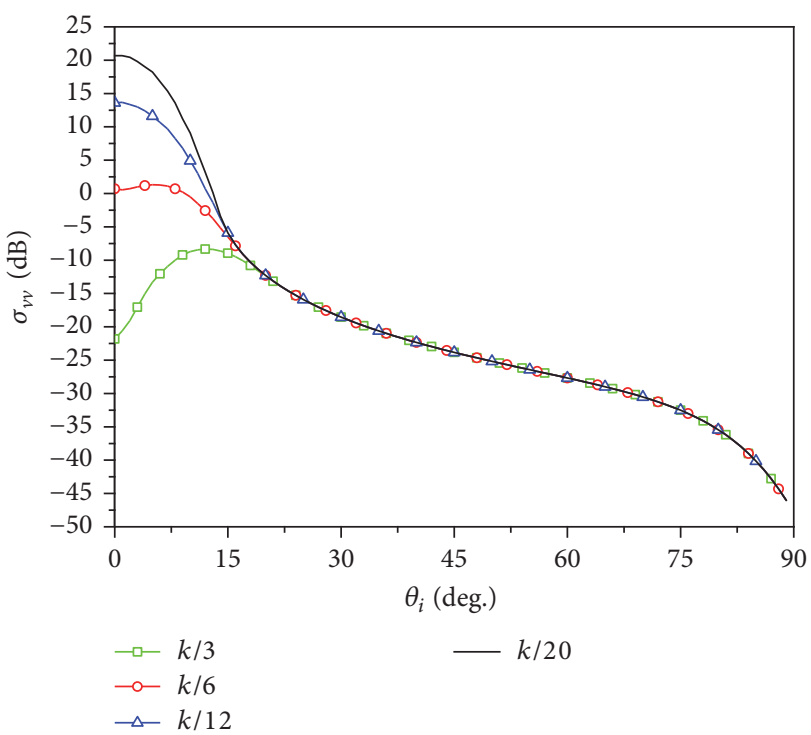

FIGURE 3: Impact of cut-off wave number on scattering coefficients.

discrete points are $M=N=256$, the distances between two adjacent points are $\Delta x=\Delta y=1.0 \mathrm{~m}$, and the relative permittivity calculated by Klein model [14] at $20^{\circ} \mathrm{C}$ and $35 \%$ salinity is $\varepsilon=(55.845,37.731)$.

In view of the dependency on cut-off wave number at near vertical incident angles, Kirchhoff model can be introduced to revise the slope-modulated perturbation coefficient [15]. Then (7) can be rewritten as

$$
\begin{aligned}
\sigma_{P Q}^{\text {total }}\left(\widehat{\mathbf{k}}_{i}, \widehat{\mathbf{k}}_{s}\right)=\frac{1}{A} \\
\cdot \sum_{m=1}^{M} \sum_{n=1}^{N}\left\{\left[\sigma_{P Q, m n}^{\mathrm{KAM}}\left(\widehat{\mathbf{k}}_{i}, \widehat{\mathbf{k}}_{s}\right)+\sigma_{P Q, m n}^{\mathrm{TSPM}}\left(\widehat{\mathbf{k}}_{i}, \widehat{\mathbf{k}}_{s}\right)\right] \Delta x \Delta y\right\},
\end{aligned}
$$

where $\sigma_{P Q, m n}^{\mathrm{KAM}}\left(\widehat{\mathbf{k}}_{i}, \widehat{\mathbf{k}}_{s}\right)$ is the normalized specular scattering coefficient of the number $m n$ facet and can be obtained by 


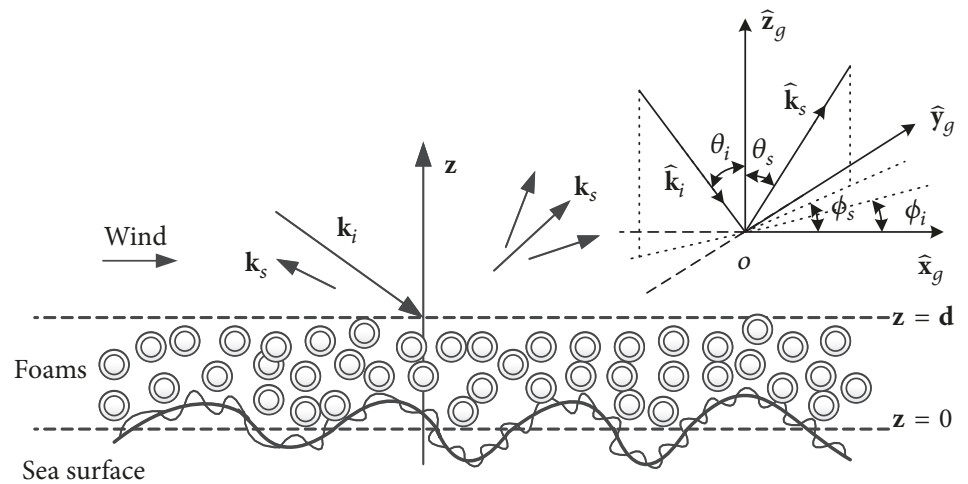

FIGURE 4: Geometry of foams scattering.

$$
\sigma_{P Q, m n}^{\mathrm{KAM}}\left(\widehat{\mathbf{k}}_{i}, \widehat{\mathbf{k}}_{s}\right)=\frac{\pi k^{2}|\mathbf{q}|^{2}}{q_{z}^{4}}\left|\widetilde{F}_{P Q}^{\mathrm{KAM}}\right| P\left(z_{x}^{\mathrm{tan}}, z_{y}^{\mathrm{tan}}\right),
$$

where $P(\bullet)$ is the slope probability density function (refer to [16]). The detailed expressions of polarization factor $\widetilde{F}_{P Q}^{\mathrm{KAM}}$ are given in Appendix.

By now, we can calculate the total scattering coefficient of a particular sea surface sample by (8). At the same time, the local contribution from any facet can be readily obtained. So this modified facet-based scattering model could be applied to SAR imagery simulation.

2.2. Foams Scattering Model. In reality, with the increase of wind speed above the sea surface, foams come into being. So it is inaccurate to calculate the scattering from sea surface with forenamed modified facet-based model and the influences of foams on sea surface scattering should be taken into account at high-wind-speed condition.

As shown in Figure 4, the Vector Radiative Transfer (VRT) functions of the foam layer can be expressed by [9]

$$
\begin{aligned}
\cos \theta_{s} & \frac{d}{d z} \bar{I}\left(\theta_{s}, \varphi_{s}, z\right) \\
= & -\overline{\overline{K_{e}}}\left(\theta_{s}, \varphi_{s}\right) \cdot \bar{I}\left(\theta_{s}, \varphi_{s}, z\right) \\
& +\int_{0}^{2 \pi} d \varphi_{i} \int_{0}^{\pi / 2} d \theta_{i} \times \sin \theta_{s} \\
& \cdot \overline{\bar{P}}\left(\theta_{s}, \varphi_{s} ; \theta_{i}, \varphi_{i}\right) \bar{I}\left(\theta_{i}, \varphi_{i}, z\right)+\bar{Q}\left(z, \theta_{s}, \varphi_{s}\right), \\
-\cos & \theta_{s} \frac{d}{d z} \bar{I}\left(\pi-\theta_{s}, \varphi_{s}, z\right) \\
= & \overline{\overline{K_{e}}}\left(\theta_{s}, \varphi_{s}\right) \cdot \bar{I}\left(\pi-\theta_{s}, \varphi_{s}, z\right) \\
& +\int_{0}^{2 \pi} d \varphi_{i} \int_{0}^{\pi / 2} d \theta_{i} \sin \theta_{s} \cdot \overline{\bar{P}}\left(\pi-\theta_{s}, \varphi_{s} ; \theta_{i}, \varphi_{i}\right) \\
& \cdot \bar{I}\left(\theta_{i}, \varphi_{i}, z\right)+\int_{0}^{2 \pi} d \varphi_{i} \int_{0}^{\pi / 2} d \theta_{i} \sin \theta_{s} \\
& \cdot \overline{\bar{P}}\left(\pi-\theta_{s}, \varphi_{s} ; \pi-\theta_{i}, \varphi_{i}\right) \cdot \bar{I}\left(\pi-\theta_{i}, \varphi_{i}, z\right) \\
& +\bar{Q}\left(z, \theta_{s}, \varphi_{s}\right),
\end{aligned}
$$

where $\theta_{i}, \emptyset_{i} \theta_{s}$, and $\emptyset_{s}$ represent incident angle, incident azimuth angle, scattering angle, and scattering azimuth angle, respectively. $\bar{I}(\theta, \varphi, z)$ is a $4 \times 1$ intensity vector. $\overline{\overline{K_{e}}}$ stands for the extinction matrix. $\bar{Q}$ indicates the heat emitter undersea which is equal to zero when illuminated by EM wave above the sea surface. $\overline{\bar{P}}$ denotes the phase matrix including the multiple scattering and coupling relationship among particles which can be obtained by Rayleigh approximation. The boundary condition is given in [10]. VRT functions could be solved utilizing boundary condition together with constant variation function, and then each order result can be obtained by iterative method.

The bistatic scattering coefficient of sea surface with foams can be defined as [17]

$$
\sigma_{p q}\left(\theta_{s}, \varphi_{s} ; \theta_{i}, \varphi_{i}\right)=\frac{4 \pi \cos \theta_{s} I_{p}\left(\theta_{s}, \varphi_{s}\right)}{I_{q}\left(\theta_{i}, \varphi_{i}\right)}
$$

where $I_{q}$ and $I_{p}$ are the incident and scattering intensities, respectively.

We can further get the zeroth-order and first-order scattering coefficients $\sigma_{p q(\mathrm{a})}^{(0)}, \sigma_{p q(\mathrm{~b})}^{(1)}, \sigma_{p q(\mathrm{c})}^{(1)}, \sigma_{p q(\mathrm{~d})}^{(1)}$, and $\sigma_{p q(\mathrm{e})}^{(1)}$ of sea surface with foams:

$$
\begin{aligned}
& \sigma_{p q(\mathrm{a})}^{(0)}\left(\theta_{s}, \phi_{s}, \theta_{i}, \phi_{i}\right)=\sigma_{p q} e^{-k_{e} d\left(1 / \cos \theta_{s}+1 / \cos \theta_{i}\right)} \\
& \sigma_{p q(\mathrm{~b})}^{(1)}\left(\theta_{s}, \phi_{s}, \theta_{i}, \phi_{i}\right)=\frac{4 \pi}{k_{e}} \cos \theta_{s} \overline{\bar{P}}_{p q}\left(\theta_{s}, \phi_{s}, \pi-\theta_{i}, \phi_{i}\right) \\
& \cdot\left(1-e^{-k_{e} d\left(1 / \cos \theta_{s}+1 / \cos \theta_{i}\right)}\right), \\
& \sigma_{p q(\mathrm{c})}^{(1)}\left(\theta_{s}, \phi_{s}, \theta_{i}, \phi_{i}\right)=\frac{4 \pi}{k_{e}} \cos \theta_{s} \int_{0}^{2 \pi} d \theta^{\prime} \sin \theta^{\prime} \\
& \cdot \int_{0}^{2 \pi} d \phi^{\prime} \sum_{l=v, h} \overline{\bar{P}}_{p l}\left(\theta_{s}, \phi_{s}, \theta^{\prime}, \phi^{\prime}\right) \\
& \cdot \overline{\bar{R}}_{p q}\left(\theta^{\prime}, \phi^{\prime}, \pi-\theta_{i}, \phi_{i}\right) \times \frac{\cos \theta^{\prime}}{\cos \theta_{s}-\cos \theta^{\prime}} \\
& \cdot e^{-k_{e} d\left(1 / \cos \theta^{\prime}-1 / \cos \theta_{s}\right)},
\end{aligned}
$$




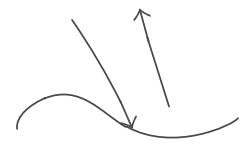

$\sigma_{p q(\mathrm{a})}^{(0)}$
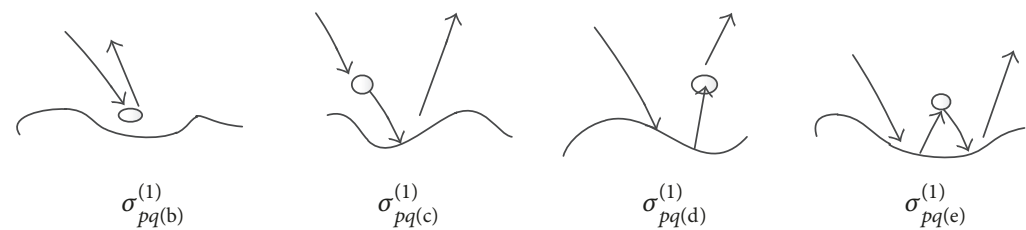

FIGURE 5: Scattering processes of different coefficients.

$$
\begin{aligned}
& \sigma_{p q(\mathrm{~d})}^{(1)}\left(\theta_{s}, \phi_{s}, \theta_{i}, \phi_{i}\right)=\frac{4 \pi}{k_{e}} \cos \theta_{s} \int_{0}^{\pi / 2} d \theta^{\prime} \sin \theta^{\prime} \\
& \cdot \int_{0}^{2 \pi} d \phi^{\prime} \sum_{m=v, h} \overline{\bar{R}}_{p m}\left(\theta_{s}, \phi_{s}, \pi-\theta^{\prime}, \phi^{\prime}\right) \\
& \times \overline{\bar{P}}_{m q}\left(\pi-\theta^{\prime}, \phi^{\prime}, \pi-\theta_{i}, \phi_{i}\right) \times \frac{\cos \theta_{i}}{\cos \theta_{i}-\cos \theta^{\prime}} \\
& \cdot e^{-k_{e} d\left(1 / \cos \theta^{\prime}-1 / \cos \theta_{i}\right)}, \\
& \sigma_{p q(\mathrm{e})}^{(1)}\left(\theta_{s}, \phi_{s}, \theta_{i}, \phi_{i}\right)=\frac{4 \pi}{k_{e}} \cos \theta_{s} \int_{0}^{\pi / 2} d \theta^{\prime} \sin \theta^{\prime} \\
& \cdot \int_{0}^{2 \pi} d \phi^{\prime} \sum_{l=v, h} \overline{\bar{R}}_{p l}\left(\theta_{s}, \phi_{s}, \pi-\theta^{\prime}, \phi^{\prime}\right) \\
& \quad \times \int_{0}^{\pi / 2} d \theta^{\prime \prime} \sin \theta^{\prime \prime} \int_{0}^{2 \pi} d \phi^{\prime \prime} \\
& \quad \times \sum_{m=v, h} \overline{\bar{P}}_{l m}\left(\pi-\theta^{\prime}, \phi^{\prime}, \theta^{\prime \prime}, \phi^{\prime \prime}\right) \overline{\bar{R}}_{m q}\left(\theta^{\prime \prime}, \phi^{\prime \prime}, \theta_{i}, \phi_{i}\right),
\end{aligned}
$$

where $k_{e}$ is the extinction coefficient and $\sigma_{p q}$ is the scattering coefficient calculated by the presented modified facet-based model above. The physical scattering processes of different coefficients are demonstrated in Figure 5.

In consequence, the scattering coefficient of the area covered with foams can be calculated by

$$
\begin{aligned}
\sigma_{p q}^{\text {foam }}\left(\theta_{s}, \varphi_{s} ; \theta_{i}, \varphi_{i}\right)= & \sigma_{p q(\mathrm{a})}^{(0)}\left(\theta_{s}, \varphi_{s} ; \theta_{i}, \varphi_{i}\right) \\
& +\sigma_{p q(\mathrm{~b})}^{(1)}\left(\theta_{s}, \varphi_{s} ; \theta_{i}, \varphi_{i}\right) \\
& +\sigma_{p q(\mathrm{c})}^{(1)}\left(\theta_{s}, \varphi_{s} ; \theta_{i}, \varphi_{i}\right) \\
& +\sigma_{p q(\mathrm{~d})}^{(1)}\left(\theta_{s}, \varphi_{s} ; \theta_{i}, \varphi_{i}\right) \\
& +\sigma_{p q(\mathrm{e})}^{(1)}\left(\theta_{s}, \varphi_{s} ; \theta_{i}, \varphi_{i}\right) .
\end{aligned}
$$

In fact, it could be assumed that the foams are made up of many water-air particles which could be represented by the model in Figure 6. Referring to [18], $a=250 \mu \mathrm{m}$ is the outer radius, $b$ is the inner radius, and $a-b=20 \mu \mathrm{m}$ is the thickness of water film. The thickness of foams here is presumed to be

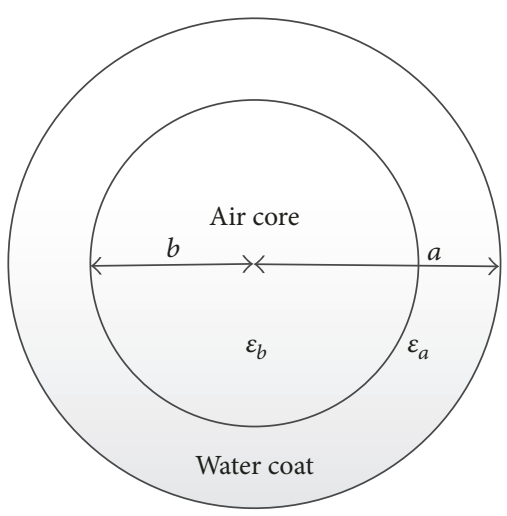

FIGURE 6: Geometry of foams.

$2 \mathrm{~cm}$. Then the extinction coefficient $k_{e}$ of this foams model can be calculated by [19]

$$
k_{e}=N_{0} \frac{2 \pi}{k^{2}} \sum_{n=1}^{\infty}(2 n+1) \operatorname{Re}\left(-T_{n}^{(N)}-T_{n}^{(M)}\right),
$$

where $N_{0}$ is the number of foams in unit volume and could be obtained according to [20].

The coverage percentage of foams in wind-driven sea surface is intimately related to wind speed and seawater temperature. While the temperature difference between air and seawater is moderate, it can be calculated by

$$
C=2.32 \times 10^{-6} U_{10}^{3.4988} .
$$

Then the number of facets covered with foams is $N_{f}=$ $M \times N \times C$. According to the slope criterion presented by Longuet-Higgins and Fox [21], we can add foams to $N_{f}$ facets with greater slopes. Thus, we can get the distribution of foams instead of the coverage percentage alone, which is of great significance to SAR imagery simulation of complex sea surface.

As a result, we can calculate the total scattering coefficient of a particular wind-driven sea surface covered with foams by

$$
\begin{aligned}
& \sigma_{p q}^{\text {total }} \\
& =\frac{\sum_{m=1}^{M N-N_{f}}\left[\left(\sigma_{p q, m}^{\mathrm{KAM}}+\sigma_{p q, m}^{\mathrm{TSPM}}\right) \Delta x \Delta y\right]+\sum_{n=1}^{N_{f}} \sigma_{p q, n}^{\mathrm{foam}} \Delta x \Delta y}{A} .
\end{aligned}
$$

\section{Model Validation and Analysis}

The accuracy and validity of the scheme proposed in this paper are verified by comparing the backward-scattering 


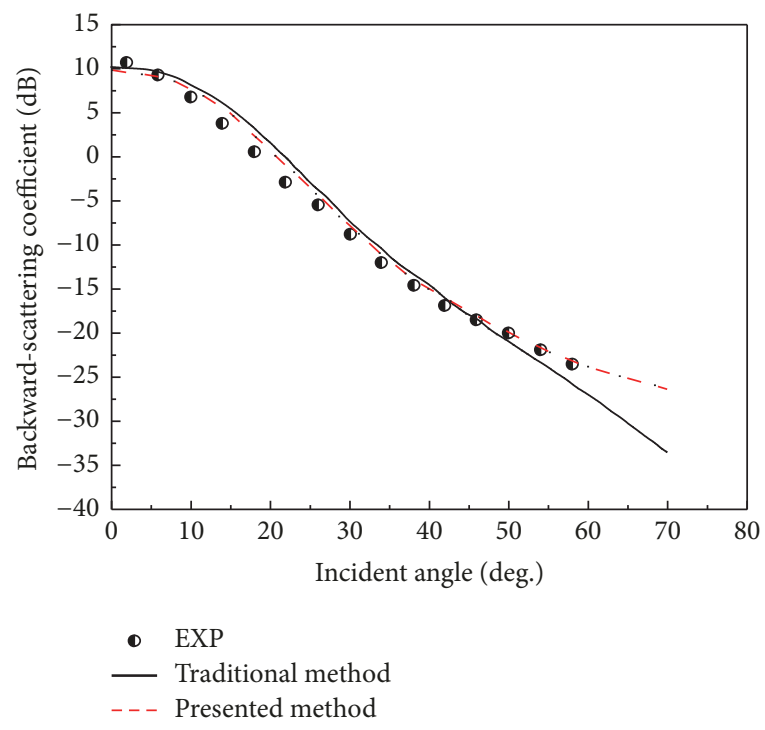

(a)

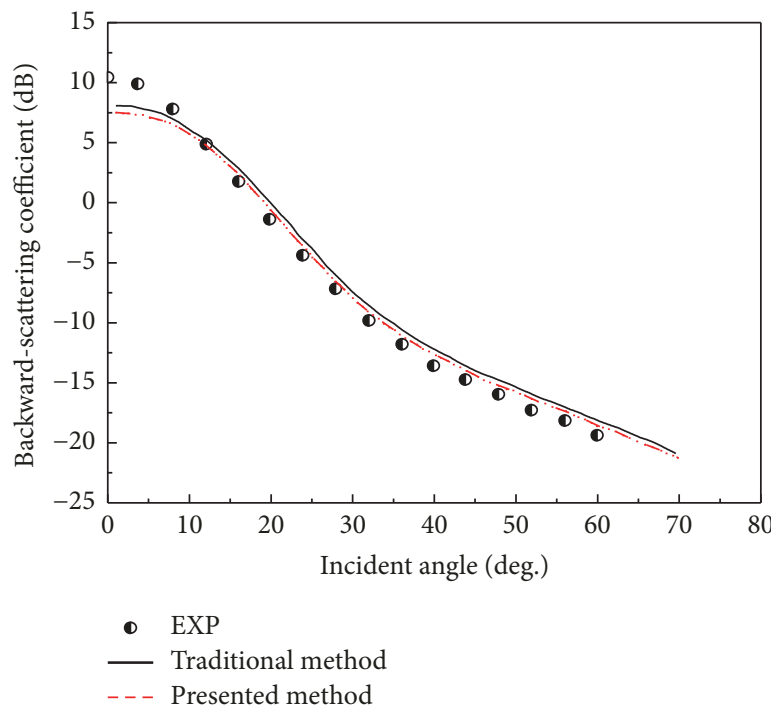

(b)

FIGURE 7: Comparison of backward-scattering results between and among the presented approach, traditional facet model, and experiment. (a) HH polarization. (b) VV polarization.

results of the presented approach with those of traditional facet model and experimental results in [22]. In simulation, the incident frequency is at $13.9 \mathrm{GHz}$, the temperature on sea surface is $17.4^{\circ} \mathrm{C}$, the salinity is $32.54 \%$, $\varepsilon=(44.43,39.55)$, and the wind speed at $10 \mathrm{~m}$ height is $10 \mathrm{~m} / \mathrm{s}$. As shown in Figure 7, the results of teh proposed approach in horizontal polarization are generally higher than those of the traditional facet model without foams, which match better with the experimental results, especially for large incidence angles. The reason for the difference is that at large incident angles the multiple scattering matters and the SPM and KA theory is unserviceable. Therefore the traditional facet model based on SPM and KA theory is inaccurate to some extent. While the foams scattering mechanism proposed in this paper includes the multiple scattering contributions which is in accordance with the physical situation, in vertical polarization, the effects of foams at different incidence angles are faint.

For further validation, Figure 8 depicts the backwardscattering results with and without foams at different wind speeds of $4 \mathrm{~m} / \mathrm{s}, 10 \mathrm{~m} / \mathrm{s}$, and $15 \mathrm{~m} / \mathrm{s}$ for vertical polarization. The incident frequency is at Ku-band. It is clearly shown that the backward-scattering results increase as the wind speed increases. This is because the sea surface becomes rougher and the incoherent scattering grows with the wind breezing up. Similarly, the influences of foams at small angles are unnoticeable and increase with the angles growing up. The differences between the backward-scattering results of sea surface with and without foams are minimum at the speed $4 \mathrm{~m} / \mathrm{s}$ and maximum at the speed $10 \mathrm{~m} / \mathrm{s}$. The reason is that the coverage rate of foams increases with the increase of wind speed and foams contribute more to the backward-scattering results.

In order to investigate the impact of azimuth angles on backward-scattering coefficients, the results at different azimuth angles are demonstrated in Figure 9. The parameters

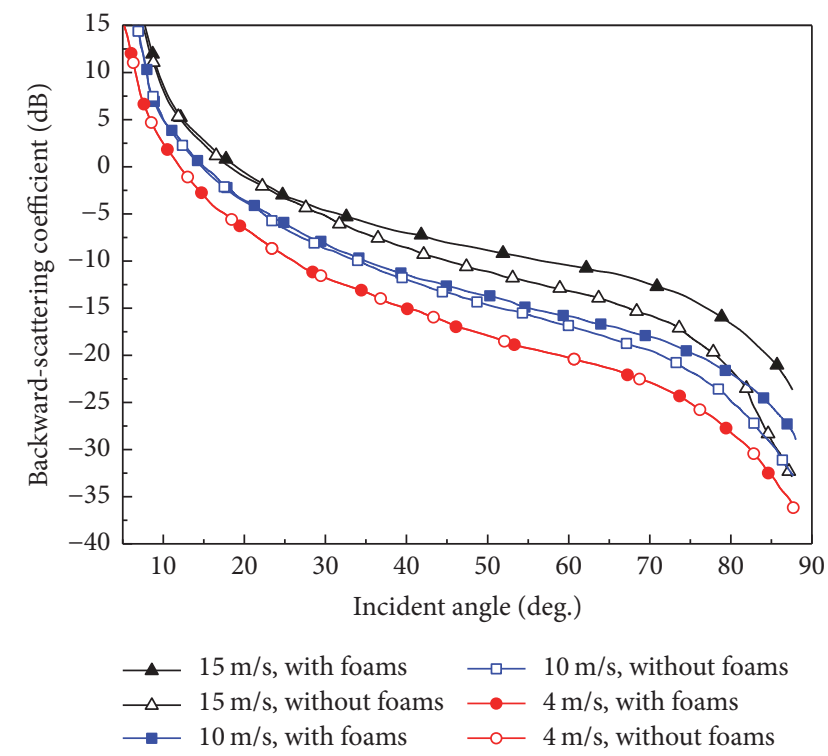

FIGURE 8: Comparison of backward-scattering results at different wind speeds.

are the same as Figure 3. It is clearly observed that the backward-scattering coefficients for vertical polarization are larger than those for horizontal polarization, while the results in horizontal polarization are more sensitive to wind speeds and azimuth angles. Furthermore, the results in downwind and backwind directions are larger than those in sidewind directions. This is because the sea surface is rougher in downwind and backwind directions than in side-wind directions, which is more noticeable at higher wind speed. In practical situation, the backward-scattering coefficients in backwind directions are always larger than those in 


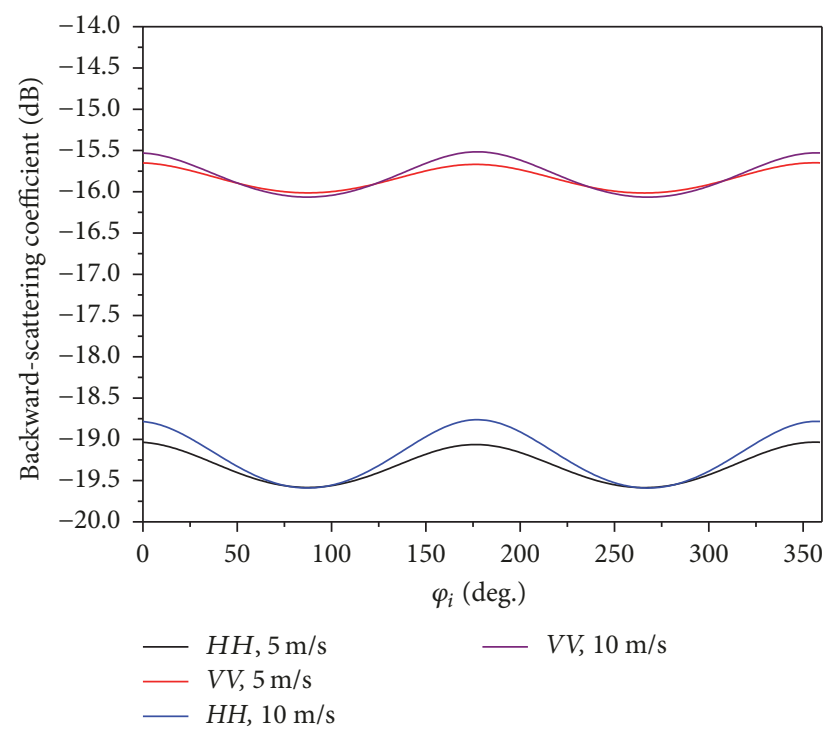

FIGURE 9: Impact of azimuth angles on backward-scattering coefficients.

downwind directions because the wave crest is tilted down the wind. All the same, the results are of no differences in simulations owing to the oversight of this asymmetry.

\section{Conclusion}

In this paper, a reliable approach based on MFBM and VRT theory is developed for the calculation of scattering from a particular electrically large sea surface covered with foams. This proposed scheme is of more comprehensive significance because the contributions from not only the specular and diffuse configurations but also the foams are taken into consideration. Moreover, it can handily give both the monostatic and bistatic scattering results and could overwhelm the application to SAR imagery simulation. The comparisons show better agreement with experimental results than traditional facet model, which demonstrates the accuracy and superiority of this scheme. Additionally, numerical results show that the contributions of foams are assignable and of great significance at large incidence angles and high wind speeds.

\section{Appendix}

The polarization factor $\widetilde{F}_{P Q}$ can be expressed as

$$
\begin{aligned}
F_{v v} & \\
= & \frac{1}{\varepsilon}\left[1+R_{v}\left(\theta_{i}\right)\right]\left[1+R_{v}\left(\theta_{s}\right)\right] \sin \theta_{i} \sin \theta_{s} \\
& -\left[1-R_{v}\left(\theta_{i}\right)\right]\left[1-R_{v}\left(\theta_{s}\right)\right] \cos \theta_{i} \cos \theta_{s} \cos \phi_{s}, \\
F_{v h}= & {\left[1-R_{v}\left(\theta_{i}\right)\right]\left[1+R_{h}\left(\theta_{s}\right)\right] \cos \theta_{i} \sin \phi_{s}, } \\
F_{h v}= & {\left[1+R_{h}\left(\theta_{i}\right)\right]\left[1-R_{v}\left(\theta_{s}\right)\right] \cos \theta_{s} \sin \phi_{s}, } \\
F_{h h}= & {\left[1+R_{h}\left(\theta_{i}\right)\right]\left[1+R_{h}\left(\theta_{s}\right)\right] \cos \phi_{s}, }
\end{aligned}
$$

where $\theta_{i}, \theta_{s}$, and $\emptyset_{s}$ are the incidence angle, scattering angle, and scattering azimuth angle, respectively; $R_{v}$ and $R_{h}$ are the Fresnel reflection coefficients in two different polarization conditions which are defined as

$$
\begin{aligned}
R_{v}= & \frac{\varepsilon \cos \theta_{i}-\sqrt{\varepsilon-\sin ^{2} \theta_{i}}}{\varepsilon \cos \theta_{i}+\sqrt{\varepsilon-\sin ^{2} \theta_{i}}}, \\
R_{h}= & \frac{\cos \theta_{i}-\sqrt{\varepsilon-\sin ^{2} \theta_{i}}}{\cos \theta_{i}+\sqrt{\varepsilon-\sin ^{2} \theta_{i}}} .
\end{aligned}
$$

The polarization factor $\widetilde{F}_{P Q}^{\mathrm{KAM}}$ can be expressed as

$$
\begin{aligned}
& \widetilde{F}_{V V}^{\mathrm{KAM}}=M_{0}\left[R_{v}\left(\theta_{i}^{l}\right)\left(\widehat{\mathbf{V}}_{s} \cdot \widehat{\mathbf{k}}_{i}\right)\left(\widehat{\mathbf{V}}_{i} \cdot \widehat{\mathbf{k}}_{s}\right)\right. \\
& \left.\quad+R_{h}\left(\theta_{i}^{l}\right)\left(\widehat{\mathbf{H}}_{s} \cdot \widehat{\mathbf{k}}_{i}\right)\left(\widehat{\mathbf{H}}_{i} \cdot \widehat{\mathbf{k}}_{s}\right)\right] \\
& \widetilde{F}_{V H}^{\mathrm{KAM}}=M_{0}\left[R_{v}\left(\theta_{i}^{l}\right)\left(\widehat{\mathbf{V}}_{s} \cdot \widehat{\mathbf{k}}_{i}\right)\left(\widehat{\mathbf{H}}_{i} \cdot \widehat{\mathbf{k}}_{s}\right)\right. \\
& \left.\quad-R_{h}\left(\theta_{i}^{l}\right)\left(\widehat{\mathbf{H}}_{s} \cdot \widehat{\mathbf{k}}_{i}\right)\left(\widehat{\mathbf{V}}_{i} \cdot \widehat{\mathbf{k}}_{s}\right)\right], \\
& \left.\quad-R_{h}\left(\theta_{i}^{l}\right)\left(\widehat{\mathbf{V}}_{s} \cdot \widehat{\mathbf{k}}_{i}\right)\left(\widehat{\mathbf{H}}_{i} \cdot \widehat{\mathbf{k}}_{s}\right)\right], \\
& \widetilde{F}_{H V}^{\mathrm{KAM}}=M_{H}\left[R_{v}\left(\theta_{i}^{l}\right)\left(\widehat{\mathbf{H}}_{s} \cdot \widehat{\mathbf{k}}_{i}\right)\left(\widehat{\mathbf{V}}_{i} \cdot \widehat{\mathbf{k}}_{s}\right)\right. \\
& \left.\quad+R_{h}\left(\theta_{i}^{l}\right)\left(\widehat{\mathbf{V}}_{s} \cdot \widehat{\mathbf{k}}_{i}\right)\left(\widehat{\mathbf{V}}_{i} \cdot \widehat{\mathbf{k}}_{s}\right)\right],
\end{aligned}
$$

where $M_{0}=|\mathbf{q}|\left|q_{z}\right| /\left\{\left[\left(\widehat{\mathbf{H}}_{s} \cdot \widehat{\mathbf{k}}_{i}\right)^{2}+\left(\widehat{\mathbf{V}}_{s} \cdot \widehat{\mathbf{k}}_{i}\right)^{2}\right] k q_{z}\right\}$.

\section{Conflicts of Interest}

The authors declare that there are no conflicts of interest regarding the publication of this paper.

\section{Acknowledgments}

This work was supported by National Natural Science Foundation of China under Grant no. 61372033.

\section{References}

[1] D. Colak, R. J. Burkholder, and E. H. Newman, "Multiple sweep method of moments analysis of electromagnetic scattering from 3D targets on ocean-like rough surfaces," Microwave and Optical Technology Letters, vol. 49, no. 1, pp. 241-247, 2007.

[2] J. T. Johnson, J. V. Toporkov, and G. S. Brown, "A numerical study of backscattering from time-evolving sea surfaces: comparison of hydrodynamic models," IEEE Transactions on Geoscience and Remote Sensing, vol. 39, no. 11, pp. 2411-2420, 2001.

[3] J. V. Toporkov and G. S. Brown, "Numerical simulations of scattering from time-varying, randomly rough surfaces," IEEE Transactions on Geoscience and Remote Sensing, vol. 38, no. 4, pp. 1616-1625, 2000. 
[4] D. D. Crombie, "Doppler spectrum of sea echo at $13.56 \mathrm{Mc} . / \mathrm{s.}$." Nature, vol. 175, pp. 681-682, 1955.

[5] F. G. Bass and I. M. Fuks, Wave Scattering from Statistically Rough Surfaces, Pergamon, Oxford, NY, USA, 1979.

[6] Z.-S. Wu, J.-P. Zhang, and L.-X. Guo, "An improved two-scale model with volume scattering for the dynamic ocean surface," Progress in Electromagnetics Research, vol. 89, pp. 39-56, 2009.

[7] G. Franceschetti, M. Migliaccio, and D. Riccio, "On ocean SAR raw signal simulation," IEEE Transactions on Geoscience and Remote Sensing, vol. 36, no. 1, pp. 84-100, 1998.

[8] H. Chen, M. Zhang, Y.-W. Zhao, and W. Luo, "An efficient slope-deterministic facet model for SAR imagery simulation of marine scene," IEEE Transactions on Antennas and Propagation, vol. 58, no. 11, pp. 3751-3756, 2010.

[9] Y.-Q. Jin, "Some results from the radiative wave equation for a slab of random, densely-distributed scatterers," Journal of Quantitative Spectroscopy \& Radiative Transfer, vol. 39, no. 2, pp. 83-98, 1988.

[10] Y. Liang and L.-X. Guo, "Study of the electromagnetic scattering from the rough sea surface with bubbles/foams by the modified two-scale method," Wuli Xuebao/Acta Physica Sinica, vol. 58, no. 9, pp. 6158-6166, 2009.

[11] I. M. Fuks, "Wave diffraction by rough interfaces in an arbitrary plane-layered medium," Waves in Random and Complex Media, vol. 10, no. 2, pp. 253-272, 2000.

[12] I. M. Fuks, "Wave diffraction by a rough boundary of an arbitrary plane-layered medium," IEEE Transactions on Antennas and Propagation, vol. 49, no. 4, pp. 630-639, 2001.

[13] T. Elfouhaily, "A unified directional spectrum for long and short wind-driven waves," Journal of Geophysical Research: Atmospheres, vol. 102, no. 7, pp. 15781-15796, 1997.

[14] L. A. Klein and C. T. Swift, "An improved model for the dielectr ic constant of sea water at mi- crowave frequencies," IEEE Transactions on Antennas and Propagation, vol. 25, no. 1, pp. 104-111, 1977.

[15] A. Arnold-Bos, A. Khenchaf, and A. Martin, "Bistatic radar imaging of the marine environment - Part I: Theoretical background," IEEE Transactions on Geoscience and Remote Sensing, vol. 45, no. 11, pp. 3372-3383, 2007.

[16] C. Cox and W. Munk, "Measurement of the roughness of the sea surface from photographs of the sun's glitter," Journal of the Optical Society of America, vol. 44, no. 11, pp. 838-850, 1954.

[17] X. Qi and X. Han, "Study of laser scattering characteristics of rough sea surface with foams," Guangxue Xuebao/Acta Optica Sinica, vol. 35, no. 8, Article ID 0829003, 2015.

[18] R. Villarino, A. Camps, and M. Vall-Llossera, "Sea foam effects on the brightness temperature at L-band," IEEE International Geoscience and Remote Sensing Symposium, pp. 3076-3078, 2003.

[19] J. D. Droppleman, "Apparent microwave emissivity of sea foam," Journal of Geophysical Research, vol. 75, no. 3, pp. 696-698, 1970.

[20] J. Wu, "Bubble flux and marine aerosol spectrum under various wind velocity," Journal of Geophysical Research, vol. 97, no. 2, pp. 2327-2333, 1992.

[21] M. S. Longuet-Higgins and M. J. H. Fox, "Measurement of breaking waves by a surface meter," Journal of Geophysical Research, vol. 88, no. C14, pp. 9823-9831, 1983.

[22] A. G. Voronovich and V. U. Zavorotny, "Theoretical model for scattering of radar signals in $\mathrm{Ku}$ - and C-bands from a rough sea surface with breaking waves," Waves in Random and Complex Media, vol. 11, no. 3, pp. 247-269, 2001. 


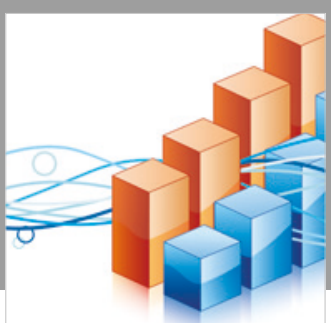

Advances in

Operations Research

\section{-n-m}
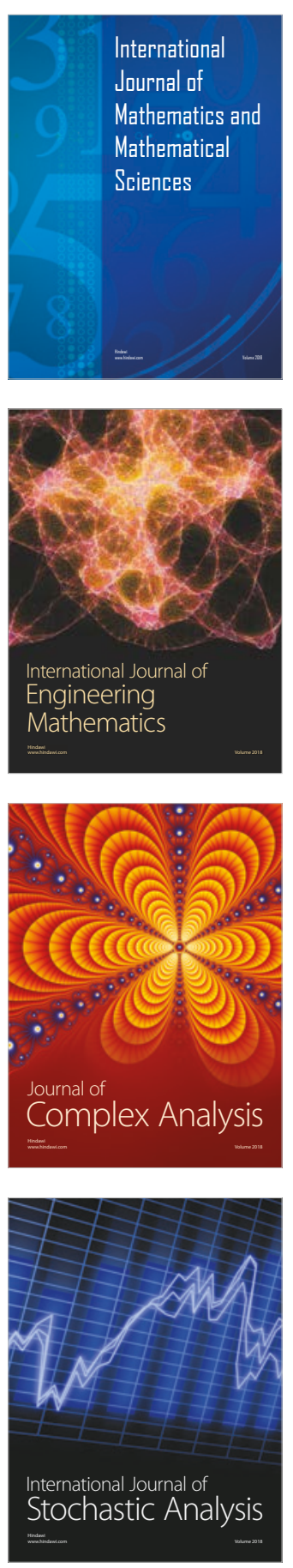
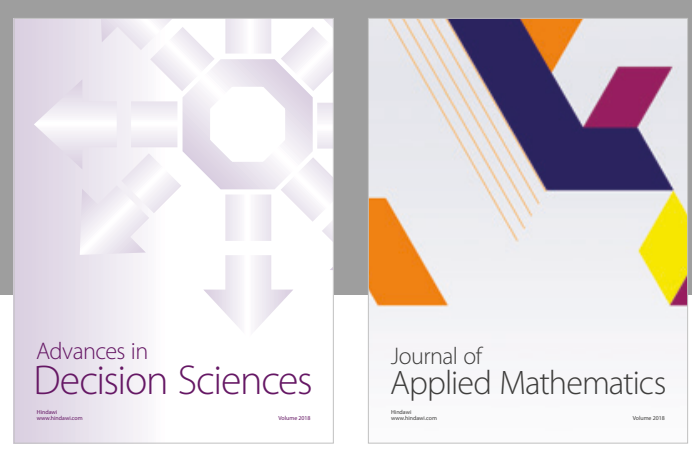

Journal of

Applied Mathematics
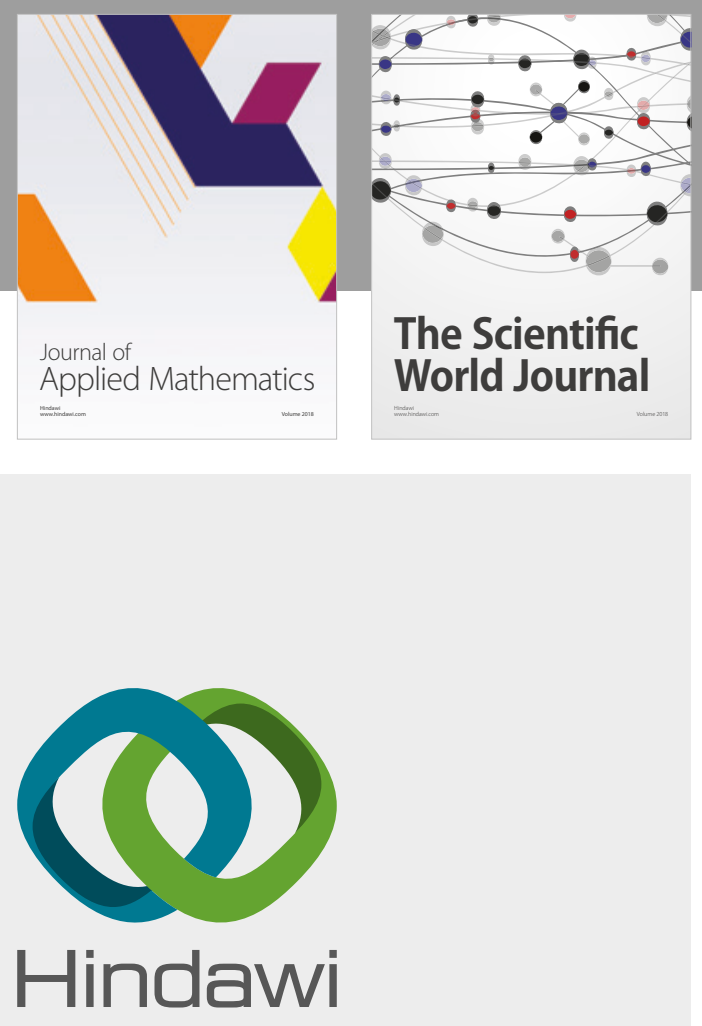

Submit your manuscripts at

www.hindawi.com

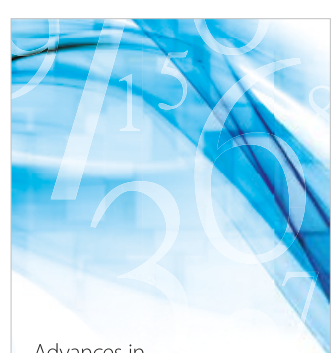

Advances in
Numerical Analysis
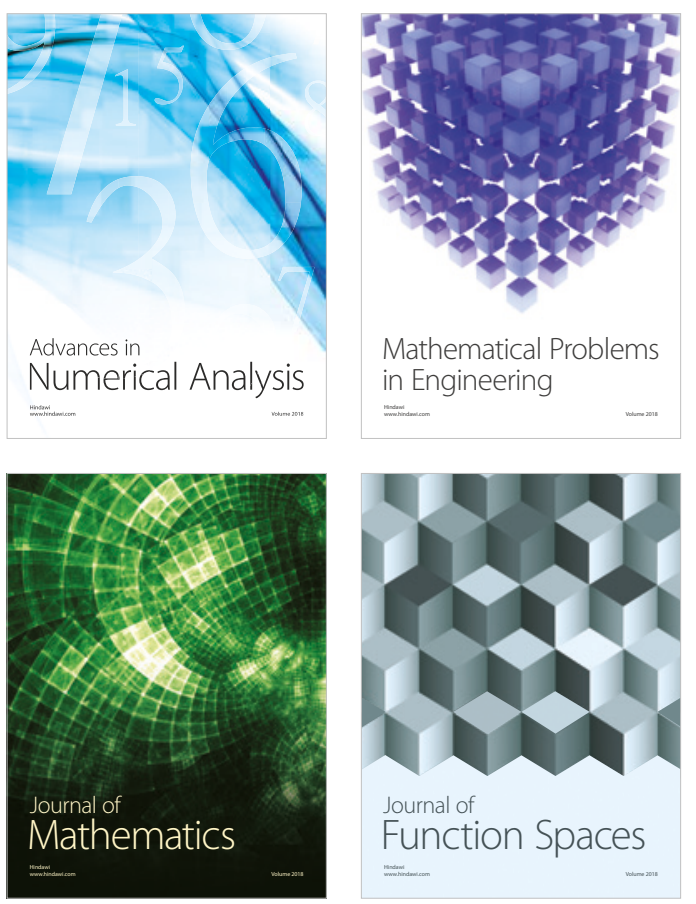

Mathematical Problems in Engineering

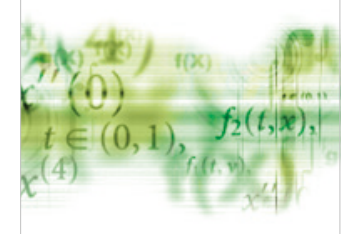

International Journal of

Differential Equations

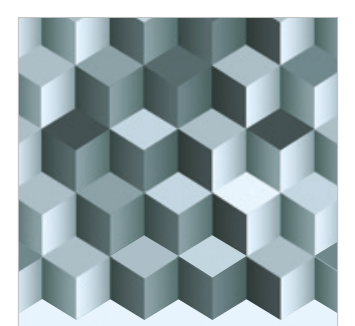

Journal of

Function Spaces

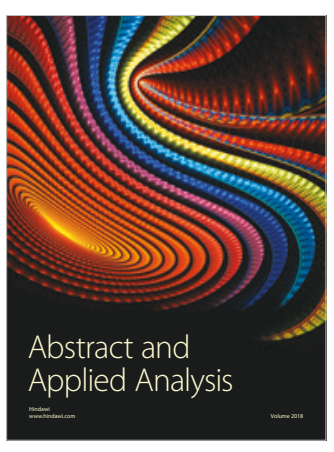

The Scientific

World Journal

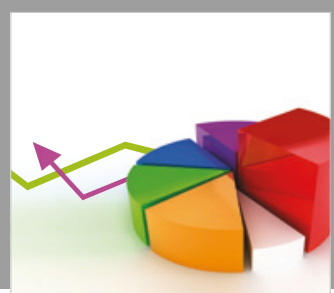

Journal of

Probability and Statistics
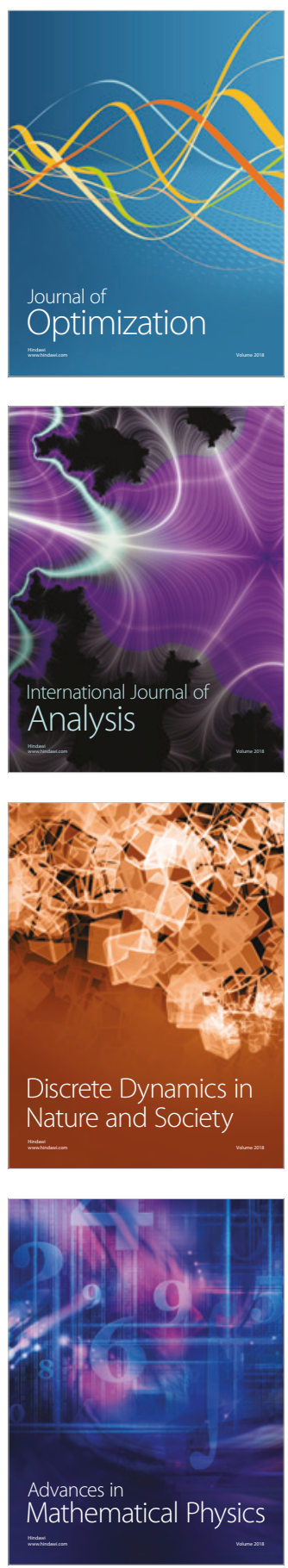